\title{
Revista d

\section{Avaliação dos níveis de chumbo e cádmio em leite em decorrência de contaminação ambiental na região do Vale do Paraíba, Sudeste do Brasil}

\section{Evaluation of lead and cadmium levels in milk due to environmental contamination in the Paraiba Valley region of Southeastern Brazil}

Isaura A. 0 kada, Alice M. Sakuma, Franca D. Maio, Sergio D ovidauskas e $\mathbf{O}$ dair Zenebon Divisão de Bromatologia e Q uímica do Instituto Adolfo Lutz. São Paulo, SP - Brasil 


\title{
Avaliação dos níveis de chumbo e cádmio em leite em decorrência de contaminação ambiental na região do Vale do Paraíba, Sudeste do Brasil*
}

\author{
Evaluation of lead and cadmium levels in milk due to \\ environmental contamination in the Paraiba Valley region \\ of Southeastern Brazil
}

Isaura A. 0 kada, Alice M. Sakuma, Franca D. Maio, Sergio Dovidauskas e $\mathbf{O}$ dair Zenebon Divisão de Bromatologia e Q uímica do Instituto Adolfo Lutz. São Paulo, SP - Brasil

Introdução

Material e Método

Resultados e Conclusões

Introduction

\section{Resumo}

Localizada em Caçapava, SP, Brasil, indústria produtora de lingotes de chumbo provocou contaminação ambiental na região do Vale do Paraíba, com chumbo e cádmio. Com o objetivo de avaliar o grau de contaminação do leite produzido na região, devido à possível ingestão, pelo gado, de gramíneas e águas contaminadas, foram determinados os teores de chumbo e cádmio no leite.

Foram analisadas 218 amostras de leite in natura e pasteurizado. O cádmio e o chumbo foram determinados por espectrofotometria de absorção atômica com chama.

Das amostras analisadas, 43 apresentaram teores de chumbo acima do limite máximo estabelecido pela legislação brasileira que é $0,05 \mathrm{mg} / \mathrm{kg}$. O valor da mediana encontrada para o chumbo foi $0,04 \mathrm{mg} / \mathrm{L}$. Os níveis de cádmio em todas as amostras foram menores que o limite de quantificação do método que é $0,02 \mathrm{mg} / \mathrm{L}$. Apesar da contaminação ambiental, os níveis encontrados para o cádmio no leite estão abaixo do limite estabelecido pela legislação brasileira que é $1,0 \mathrm{mg} / \mathrm{kg}$.

Cádmio, análise. Chumbo, análise. Leite, química.

\begin{abstract}
s
A factory producing lead ingots, located in Caçapava, caused lead and cadmium contamination of the environment, in the Paraiba Valley region of Southeastern, Brazil, through the discharge of industrial waste and the recycling of batteries. The factory, set in a rural, dairy cattle breeding area, worried sanitary authorities who envisaged the possibility of these metals' having entered the food chain. For the purpose of assessing the levels of contamination of the milk produced in the region, due to the cattle's possible consumption of contaminated grass and water, the amounts of cadmium and lead present in the milk were verified.
\end{abstract}

\footnotetext{
* Trabalho apresentado no IV Encontro Nacional sobre Contaminantes Inorgânicos em São Paulo, 1994.

Correspondência para / Correspondence to: Alice M. Sakuma, Seção de Equipamentos Especializados do Instituto Adolfo Lutz. Av, Dr. Arnaldo, 355 - 01246-902 São Paulo, SP - Brasil. 
Material and Method

\section{Results and Conclusions}

\begin{abstract}
Major producers, covering an area of up to $20 \mathrm{~km}$ from the contaminated source, authorized collection of 218 samples of both pasteurized and non-pasteurized milk, which were analysed. Lead and cadmium levels were determined by flame atomic absorption spectrophotometry, the lead being pre-concentrated by complexation with APDC (ammonium 1-pyrrolidinecarbodithioate) and further extraction with isobutyl methylketone.

Of the total number of samples, 43 presented lead levels over the maximum limit of $0.05 \mathrm{mg} / \mathrm{kg}$ estabilished by Brazilian legislation. The median value found for lead was $0.04 \mathrm{mg} / \mathrm{L}$. The variance analysis, with $95 \%$ confidence level, found no significant difference among the types of milk studied with regard to lead levels. As for cadmium, all samples showed levels below the 0.02 $\mathrm{mg} / \mathrm{L}$ quantification limit of the method. In spite of the environmental contamination, the levels of cadmium found in the milk were below the $1.0 \mathrm{mg} / \mathrm{kg}$ limit established by Brazilian legislation.
\end{abstract}

Cadmium, analysis. Lead, analysis. Milk, chemistry.

\section{INTRO DUÇÃO}

O leite, alimento amplamente consumido, principalmente por crianças, deve apresentar qualidade adequada para assegurar a saúde da coletividade. Para isto, é necessário que o leite seja proveniente de animais sadios, colhido e armazenado em condições sanitárias satisfatórias e livre de contaminação ambiental. De acordo com a legislação do Ministério da Agricultura ${ }^{3}$, todo vasilhame empregado no acondicionamento de leite, na ordenha, na coleta ou para mantê-lo em depósito deve ser de aço inoxidável, alumínio ou ferro estanhado, de perfeito acabamento e sem falhas.

Na literatura científica são relatados vários casos de intoxicação de bovinos por exposição ambiental ou ingestão de ração contaminada com chumbo e cádmio $^{2,9,15,18,19}$. Sharma e col. ${ }^{18}$ observaram que a ingestão, pelos animais, de quantidades maiores que $500 \mathrm{mg}$ de chumbo por dia ocasionou um aumento nos níveis de chumbo no leite, atingindo valores cerca de $0,06 \mathrm{mg} / \mathrm{L}$. Para o cádmio, a ingestão diária de $40 \mathrm{mg}$ não proporcionou elevação nos níveis desse metal no leite.

Devido à elevada toxicidade do chumbo e do cádmio, mesmo em nível de traços, as autoridades sanitárias mundiais estão preocupadas em estabelecer medidas para reduzir a concentração desses metais nos alimentos, uma vez que são considerados a principal fonte de ingestão desses contaminantes. Nos Estados Unidos existe campanha junto às indústrias alimentícias para conscientizar da necessidade de restringir esta contaminação e preservar a saúde coletiva. Observou-se que os níveis de chumbo vêm diminuindo significativamente em função da substi- tuição gradativa dos tipos de embalagens e soldas que posssam liberar chumbo durante $\mathrm{o}$ armazenamento dos alimentos ${ }^{17}$.

Em crianças, a absorção do chumbo e cádmio ingeridos é maior que nos adultos ${ }^{19} \mathrm{e}$ depende do tipo da dieta alimentar e do estado nutricional do indivíduo $^{12,14}$. O efeito tóxico em crianças é maior por que o chumbo depositado nos ossos fica em constante mobilidade devido ao seu crescimento. $\mathrm{O}$ esqueleto, na primeira infância, aumenta quarenta vezes em relação à sua massa original, e durante esse período há uma maior capacidade de acumular chumbo ${ }^{15,}$ 16, 17 . Ziegler e col. ${ }^{19}$ mostraram que as crianças absorvem cerca de $40 \%$ do chumbo ingerido, enquanto que os adultos absorvem somente 5 a $10 \%$.

Em 1972, o "Joint Expert Committee on Food Additives" estabeleceu como ingestão semanal tolerada provisória (ISTP) de chumbo o valor de $50 \mu \mathrm{g}$ por kg de peso corpóreo, para adultos, e $25 \mu \mathrm{g}$ por kg de peso corpóreo, para crianças; em 1993, adotou para todas as faixas etárias a ISTP de $25 \mu \mathrm{g}$ por $\mathrm{kg}$ de peso corpóreo. No caso do cádmio, em 1988, foi estabelecido o valor de $7 \mu \mathrm{g}$ por kg de peso corpóreo, para a ingestão semanal provisória tolerada, que foi ratificado em $1993^{11}$.

Uma indústria, localizada em Caçapava, SP, desde 1979, produtora de lingotes de chumbo, através da reciclagem de resíduos industriais e de baterias, provocou contaminação ambiental na região do Vale do Paraíba, com chumbo e cádmio ${ }^{6}$. A Companhia de Tecnologia de Saneamento Ambiental (CETESB), em 1993 efetuou um programa de monitorização ambiental na citada região e detectou a presença de chumbo e cádmio em amostras de sedimentos, água e gramíneas. Nessas o teor de cádmio 
encontrado foi de $1,5 \mathrm{mg} / \mathrm{kg}$, que é $50 \%$ maior que o valor normal, enquanto que para o chumbo os níveis encontrados foram até 300 vezes superiores aos considerados normais ${ }^{6}$. A localização desta indústria em área rural, que desenvolve a pecuária leiteira, causou preocupação às autoridades sanitárias devido à possibilidade daqueles metais entrarem na cadeia alimentar.

Com o objetivo de avaliar o grau de contaminação do leite produzido na região, devido à possível ingestão, pelo gado, de gramíneas e águas contaminadas, foram determinados os teores de chumbo e cádmio em amostras de leite in natura e pasteurizado, utilizando a técnica da espectrofotometria de absorção atômica com chama.

\section{MATERIAL E MÉTO DO}

Foi utilizado o espectrofotômetro de absorção atômica, marca Perkin Elmer, modelo 1100 B, equipado com corretor de deutério e lâmpadas de catodo oco de cádmio e chumbo.

As vidrarias, após lavagem com água e detergente, foram descontaminadas durante $48 \mathrm{~h}$ utilizando uma solução de $\mathrm{HNO}_{3}$ a $20 \%$ em água, e posteriormente foram enxagüadas com água destilada e desmineralizada e secas em estufa.

As amostras foram coletadas pela Vigilância Sanitária nos principais sítios produtores, num raio de $20 \mathrm{~km}$ de distância da fonte poluidora.

Foram analisadas 218 amostras de leite, sendo 139 in natura e 79 pasteurizados, dos quais 9 do tipo A, 20 do tipo B e 50 do tipo C, de diferentes marcas, produzidos na região do Vale do Paraíba, Estado de São Paulo, no período de maio a agosto de 1994.

$\mathrm{O} \mathrm{Cd}$ e o $\mathrm{Pb}$ foram determinados por espectrofotometria de absorção atômica com chama, sendo que o chumbo foi pré-concentrado por complexação com APDC (pirrolidina ditiocarbamato de amônio) e posterior extração. Foi utilizado o método da "Association of Official Analytical Chemists 1 ", substituindo o acetato de butila por metil isobutil cetona na extração.

\section{RESU LTAD O $S$}

Todas as amostras foram analisadas em duplicata, e os resultados referem-se à média de duas determinações.

Os níveis de cádmio em todas as amostras foram menores que o limite de quantificação do método $(0,02 \mathrm{mg} / \mathrm{L})$.

Os valores da média, mediana, mínimo e máximo de chumbo nas 218 amostras de leite analisadas foram, respectivamente: 0,$05 ; 0,04 ; 0,01$ e $0,20 \mathrm{mg} / \mathrm{L}$.

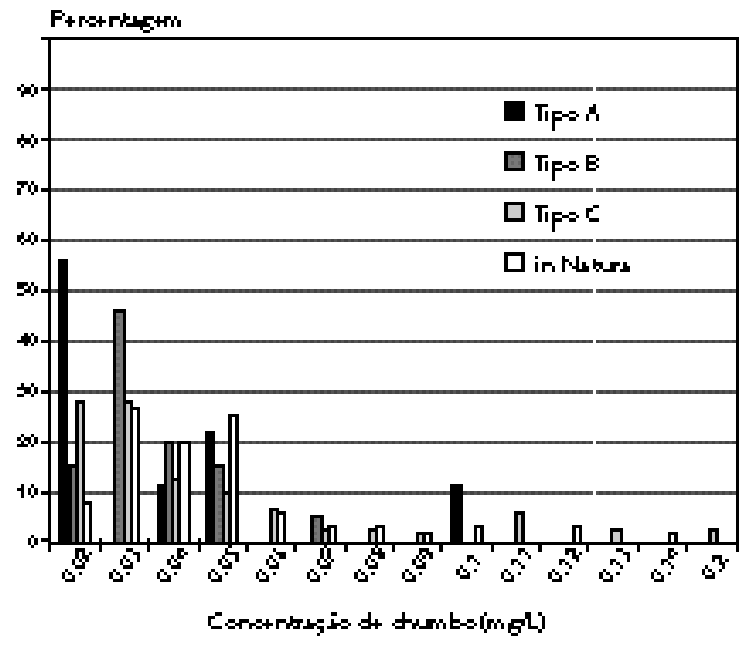

Figura - Distribuição percentual dos níveis de chumbo nos diferentes tipos de leite.

Figure - Percentual distribution of lead levels in different types of milk.

Na Figura são apresentadas as freqüências percentuais e as concentrações de chumbo para os diferentes tipos de leite.

Para o estudo da exatidão do método foi usado um material de referência certificado de leite em pó "Food Analysis Performance Assessment Scheme" - FAPAS). A recuperação foi de $96 \%$ para o chumbo e $84 \%$ para o cádmio, com um desvio-padrão relativo de $2,9 \%$ para o $\mathrm{Pb}$ e $8,6 \%$ para o $\mathrm{Cd}$.

Para o cálculo dos limites de detecção e de quantificação foram utilizadas as definições da IUPAC ${ }^{13}$. Para o chumbo e o cádmio os limites de detecção foram, respectivamente, $0,01 \mathrm{e} 0,004 \mathrm{mg} / \mathrm{L}$, e os de quantificação foram, respectivamente, $0,027 \mathrm{e}$ $0,02 \mathrm{mg} / \mathrm{L}^{13}$.

\section{DISCU SSÃO E CON CLU SÃO}

Apesar da contaminação ambiental, os níveis encontrados para o cádmio no leite estão abaixo do limite estabelecido pela legislação brasileira que é de $1,0 \mathrm{mg} / \mathrm{kg}^{4}$. Os teores de cádmio em leite encontrados pelos países participantes do Programa de Monitorização de Alimentos da Organização Mundial da Saúde, que tem por objetivo primordial coletar, avaliar e disseminar informações a respeito dos contaminantes em alimentos e o significado para a Saúde Pública ${ }^{8}$, são comparáveis aos níveis encontrados no presente estudo.

A mediana dos valores encontrados para o chumbo nas amostras analisadas foi cerca de 30 vezes maior que o encontrado por Dabeka e McKenzie ${ }^{7}$ 
em leites de vaca comercializados no Canadá. Das amostras analisadas, $20 \%$ apresentaram teores de chumbo acima do limite estabelecido pela legislação brasileira ${ }^{5}$, que é de $0,05 \mathrm{mg} / \mathrm{kg}$. Pela análise de variância com $95 \%$ de confiança verificou-se que não há diferença significativa entre os vários tipos de leites estudados.

No Brasil, em 1990, o Ministério da Saúde reviu os níveis de tolerância para chumbo em alimentos; diminuiu os níveis de aceitabilidade de chumbo de $8,0 \mathrm{mg} / \mathrm{kg}$ para $0,8 \mathrm{mg} / \mathrm{kg}$ para a maioria dos alimentos, sendo que para o leite o nível é de $0,05 \mathrm{mg} / \mathrm{kg}^{5}$. A nossa legislação apresenta o mesmo limite da legislação holandesa. Na Dinamarca e Alemanha a legislação é mais restritiva, com limites de 0,02 e $0,03 \mathrm{mg} / \mathrm{kg}$ respectivamente ${ }^{10}$.

\section{REFERÊN CIAS BIBLIO G RÁFICAS}

1. ASSOCIATION OF OFFICIAL ANALYTICAL CHEMISTS. Official methods of analysis of the Association of Official Analytical Chemists. $1^{\text {th }}$ ed. Washington, D. C., A.O.A.C., 1990. p. 255. (Tecn. 97.335).

2. BAARS, A.J. et al. Lead intoxication in cattle: a case report. Food Addit. Contam., 9:357-64, 1992.

3. BRASIL. Leis, etc. Regulamento da inspeção individual e sanitária de produtos de origem animal: aprovado pelo decreto $n^{\circ} 30.391$ de 29 de março de 1952 alterado pelo decreto $n^{\circ} 1255$, de 25 de julho de 1962. Brasília, Ministério da Agricultura, 1980.

4. BRASIL. Leis, etc. Decreto $n^{\circ} 55.871$ de 26 de março de 1965. Diário Oficial da União, Brasília, 9 abr. 1965. Seção 1, pt. 1, p. 3611.

5. BRASIL. Leis, etc. Portaria ${ }^{\circ} 16$ de 13 de março de 1990 : fixa limites máximos de tolerância de chumbo em alimentos. Diário Oficial da União, Brasília, 15. mar. 1990. Seção I, p. 5436

6. COMPANHIA DE TECNOLOGIA DE SANEAMENTO AMBIENTAL (CETESB). Contaminação ambiental por chumbo em torno da indústria FAÉ S.A. - Indústria e Comércio de Metais. São Paulo, 1993.

7. DABEKA, R.W. \& MC KENZIE, A.D. Lead and cadmium levels in commercial infant foods and dietary intake by infants 0 - 1 year old. Food Addit. Contam., 5:333-42, 1988.

8. GALAL-GOCHEV, H. Dietary intake of pesticide residues: cadmium, mercury and lead. Food Addit. Contam., 8:793806, 1991.

9. INTERNATIONAL DAIRY FEDERATION. Metal contaminants in milk and milk products. Bull. Int. Dairy Fed. (105):27-32, 1978.
Apesar do teor elevado de chumbo nas gramíneas, detectado pela CETESB $^{6}$, os leites da região do Vale do Paraíba não apresentaram valores muito elevados desse metal, o que deixou de provocar intoxicação aguda na população da região, principalmente em crianças que são as maiores consumidoras de leite. Os efeitos tóxicos do chumbo poderão surgir ao longo do tempo se medidas corretivas urgentes não forem tomadas nos processos produtivos das indústrias da região.

Para se evitar novos impactos à saúde coletiva é imperativa a ampliação de programas de monitorização da qualidade do leite produzido na região quanto à contaminação por chumbo. Deve-se também, ressaltar a importância de uma vigilância constante junto às indústrias poluidoras do meio ambiente.

10. INTERNATIONAL DAIRY FEDERATION. Trace elements in milk and milk products. Bull. Int. Dairy Fed. (278):31-9, 1992.

11. JOINT FAO / WHO EXPERT COMMITTEE ON FOOD ADDITIVES, $41^{\text {a }}$, Geneva, 1993. Toxicological evaluation of certain food additives and contaminants. Geneva, WHO/IPCS, 1993. (WHO Food Additives Series, 32).

12. JORHEM, L. \& SLORACH, S. Lead, chromium, tin, iron and cadmium in foods in welded cans. Food Addit. Contam., 4:309-16, 1987.

13. LONG, G. L. \& WINEFORDNER, J. D. Limit of detection: a closer look at the IUPAC definition. Anal. Chem., 55:712A-24A, 1983.

14. MAHAFFEY, K. R. Environmental lead toxicity: nutrition as a component of intervention. Environ. Health Perspect., 89:75-8, 1990.

15. MURTA, P.H.G.; GERMANO, M.I.S.; MIGUEL, O.; GERMANO, P.M.L. A influência da poluição ambiental sobre a qualidade do leite. Hig. Aliment., 7:12-4, 1993.

16. CENTRO PANAMERICANO DE ECOLOGIA HUMANA Y SALUD/ORGANIZACION PANAMERICANA DE LA SALUD. Evaluación epidemiologica de riesgos causados por agentes químicos ambientales. México D. F., Editorial Limusa, 1988.

17. SCHAFFNER, R.M. Lead in canned food. Food Technol., 60-4, Dec., 1981.

18. SHARMA, R.P.; STREET, J.C.; SHUPE, J.L.; BOURCIER, D.R. Accumulation and depletion of cadmium and lead in tissues and milk of lactating cows fed small amounts of these metals. J. Dairy Sci., 65:972-9, 1982.

19. ZIEGLER, E.E.; EDWARDS, B.B.; JENSEN, R.L.; MAHAFFEY, K.R.; FOMON, S.J. Absorption of lead by infants. Pediatrics, 12:29-34, 1978. 